\title{
An Indoor Positioning Algorithm Using Bluetooth Low Energy RSSI
}

\author{
Song Chai ${ }^{*}$, Renbo An and Zhengzhong Du \\ College of Electrical \& Information Engineering, Southwest University for Nationalities, Chengdu, China \\ *Corresponding author
}

\begin{abstract}
As the Bluetooth technology evolves to its 4.0 version, great applicational opportunities emerge based on the inquiry of Received Signal Strength Index (RSSI). In this paper, a positioning algorithm using Bluetooth Low Energy RSSI is proposed for indoor application. First in our algorithm, RSSI value is pre-processed: outliers of RSSI are removed, and moving average of RSSI is calculated. Then distance is determined using pre-processed RSSI and Kalman filtering. Finally, a triangulation algorithm is used to calculate the current location of the mobile device. Experiment results show that our algorithm achieves positioning accuracy of $0.2 \sim 0.5 \mathrm{~m}$.
\end{abstract}

Keywords-bluetooth low energy; RSSI; indoor positioning

\section{INTRODUCTION}

With the rapid development of wireless technology, today's market is blooming with positioning /navigation applications [1]. Satellite based positioning system such as GPS, Beidou and GLONASS provide accurate positioning service in outdoor environment. However, due to weak signal reception, these positioning system cannot work in the indoor scenario [2].

Most indoor positioning are implemented based on Received Signal Strength Index (RSSI) [3]. Mobile device receives signal from wireless node (or beacon) with known location. The distance between mobile device and beacon are calculated using RSSI of received signal. Once mobile device knows the distance between three beacons, its coordinates are triangulated.

In this paper, an algorithm is proposed for indoor positioning based on Bluetooth Low Energy RSSI. In our algorithm, RSSI is first pre-processed using single-direction outlier removal and moving average. Then a distance is calculated and filtered with Kalman filter. Finally, location is determined using triangulation.

The rest of this paper is organized as follow: section 2 summarizes some related work in indoor positioning; section 3 describe our positioning algorithm in detail; section 4 shows the results of our algorithm; section 5 concludes the paper.

\section{RELATED WORK}

Current indoor positioning methods are mainly using WIFI or Bluetooth technology [4]. In [5], Wi-Fi Fingerprint based localization with k-Nearest Neighbor algorithm has been used for indoor navigation application. An extended Kalman filter is proposed in [6] for WIFI based indoor positioning. [7] proposed an indoor positioning algorithm using iBeacon. A stigmergic approach is proposed for indoor localization Bluetooth Low Energy in [8].

\section{A. Proposed Algorithm}

1) Pre-process of RSSI value: The received RSSI are unstable even in a well-controlled indoor scenario due to multipath fading [9]. Figure I shows the received RSSI that beacon is $1 \mathrm{~m}$ away and facing directly to the mobile device. As shown in the figure, RSSI varies vigorously from $-61 \mathrm{~dB}$ to $-80 \mathrm{~dB}$. Thus outliers of RSSI needs to be removed before any further process.

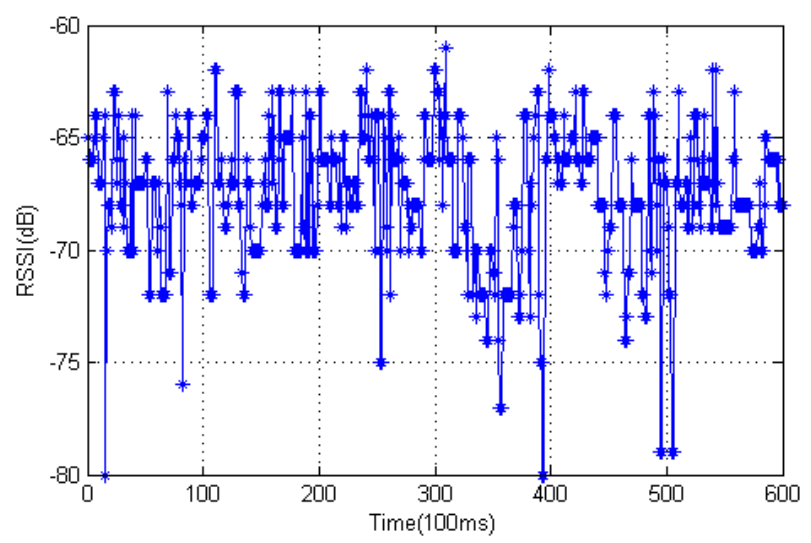

FIGURE I. RECEIVED RSSI VALUES IN 1M DISTANCE

In our algorithm, a single direction outlier removal is applied to the received RSSI, for the reason that RSSI have a tendency to decline due to indoor multipath fading. Ten recent RSSI values are stored, and mean (rssi_mean) and standard deviation (rssi_std) of these 10 RSSI are calculated. Any RSSI that is below (rssi_mean $\left.-2 * r s s i \_s t d\right)$ is removed from the stored RSSI. Then the average value of the remaining RSSI, $r s s i \_p$, is the pre-processed RSSI and used in future process.

2) Kalman Filtering: Distance between beacon and cellphone is calculated using pre-processed RSSI and Eq. 1:

$$
\boldsymbol{d i s t}= \begin{cases}10^{\frac{r s s i_{-} p}{r s a l i}}, & r s s i_{-} p>r s s i_{-} c a l i . \\ 0.9 \times 7.71^{\frac{r s s i_{-} p}{r s i_{-} c a l i}}+0.11, & r s s i_{-} p<r s s i_{-} c a l i .\end{cases}
$$

Where $r s s i \_p$ is the pre-processed RSSI and rssi_cali is the calibration RSSI which is the RSSI measured at $1 \mathrm{~m}$ distance. 


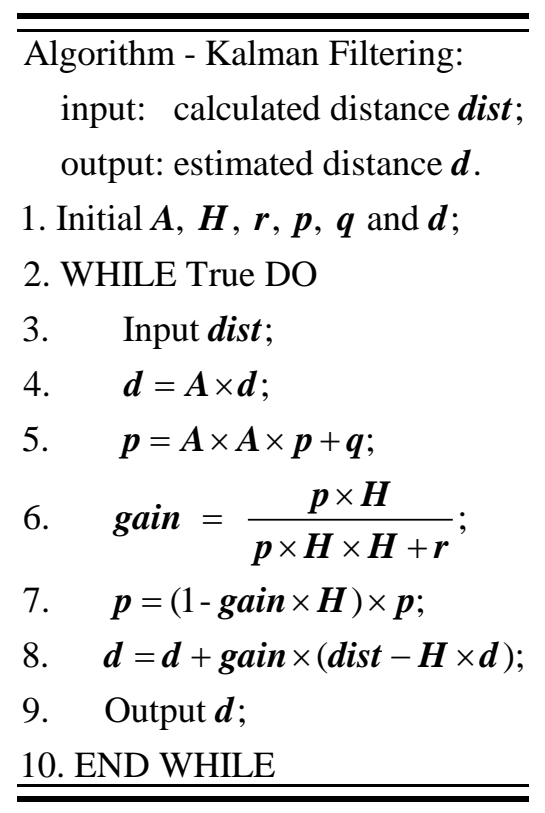

FIGURE II. KALMAN FILTERING

Then actual distance $d$ is estimated using calculated distance dist and Kalman filtering. The process of Kalman filtering is shown in Figure II.

3) Triangulation: Once mobile device knows distance from three known beacon, triangulation is performed to determine its coordinates. Three circles, centered at each beacon with radius equals to the distance between each beacon and mobile device are drawn in Figure III. The triangulation location is the centroid of the triangle $\mathrm{ABC}$, which is consisted of cords of the intersection part of three circles.

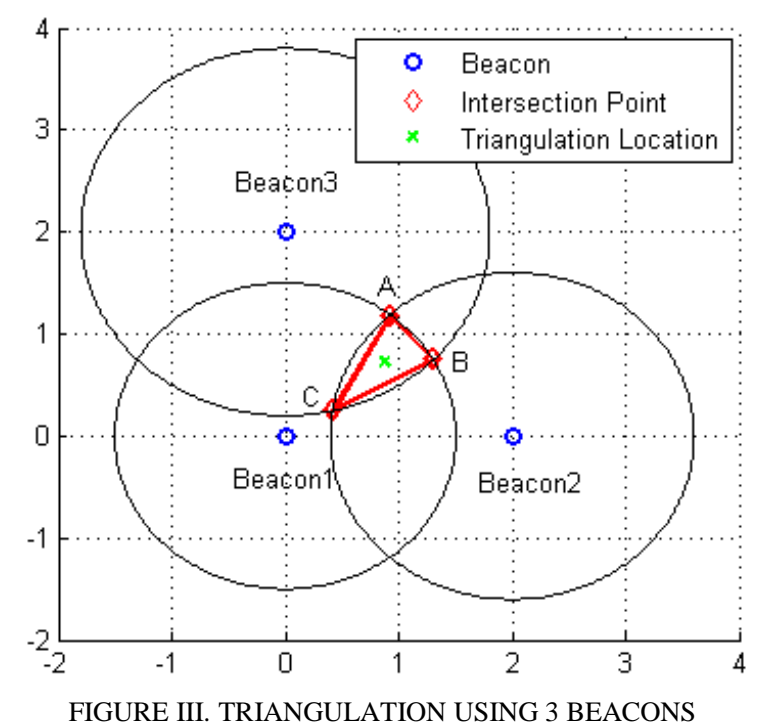

\section{B. Experiments \& Results}

Our positioning algorithm was implemented in Java using the Android SDK and run on Huawei B199 cellphone. Bluetooth beacons used in our test are AprilBeacon 241 [10].
Figure IV shows the result of pre-processed RSSI, where received RSSI is measured by facing mobile device directly to the beacon in $1 \mathrm{~m}$ distance. The received RSSI has variance of $9.86 \mathrm{~dB}$, where pre-processed RSSI only has variance of $3.35 \mathrm{~dB}$. As observed in the figure, pre-processed RSSI has less spikes in the data curve.

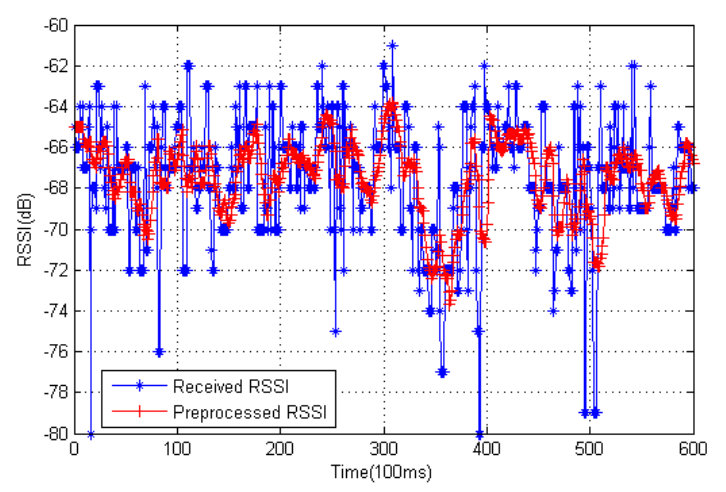

FIGURE IV. PRE-PROCESSED RSSI

A scene is setup to test the positioning accuracy of our algorithm. In a room, four beacons are placed in coordinates $(0$, $0),(4.2,0),(0,3.9)$ and $(4.2,3.9)$. The unit of coordinates are meter. Then the mobile device is place in four different location, $(2.6,1.3),(2.2,3.0),(1.6,1.4)$ and $(2.3,1.7)$, and performs our positioning algorithm. In each location, mobile device records 600 calculated coordinates. The positioning results, average calculated locations and average positioning error are illustrated in Figure V (a) (d).

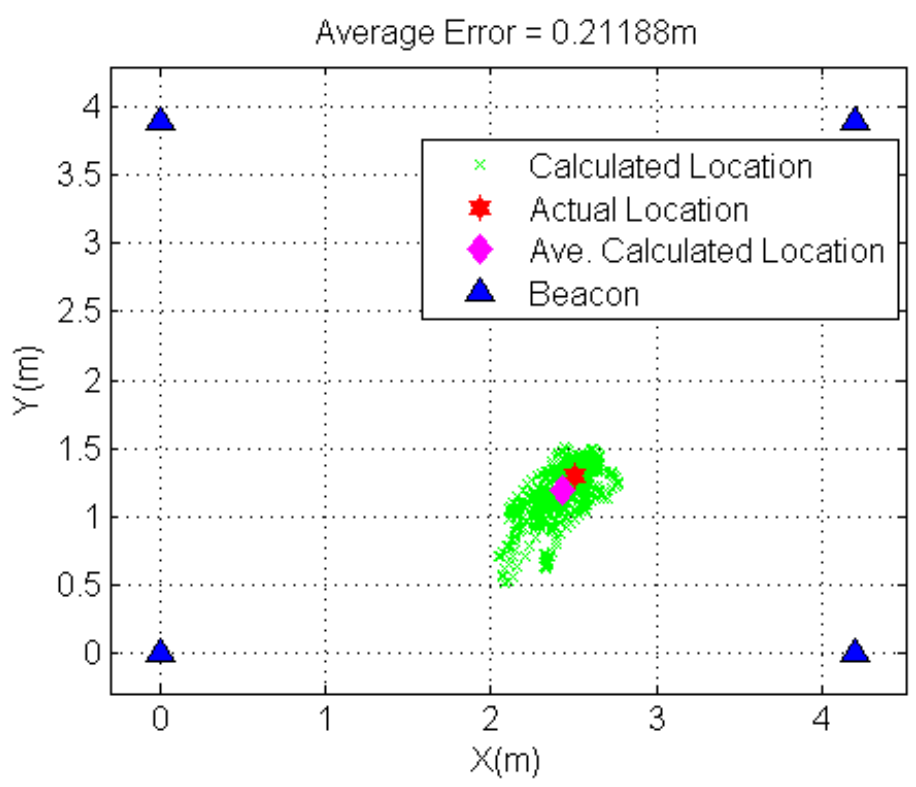

(a) Mobile device located at $(2.6,1.3)$ 


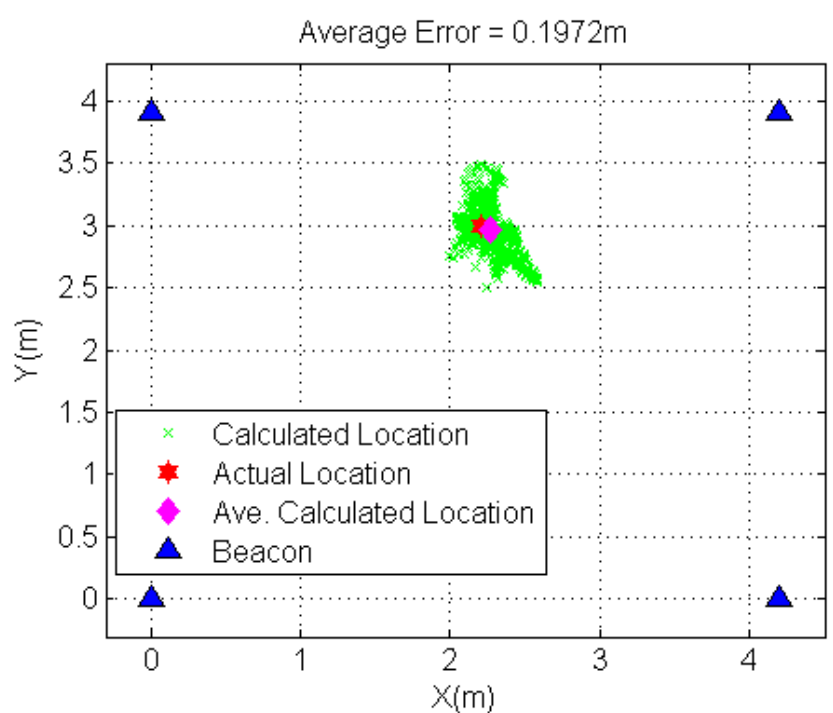

(b) Mobile device located at $(2.2,3.0)$ Average Error $=0.24521 \mathrm{~m}$

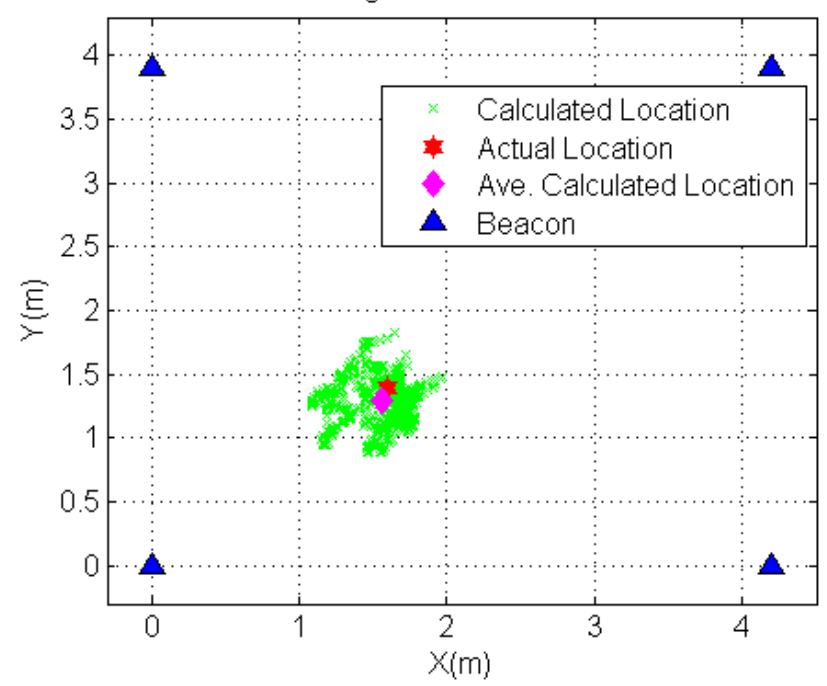

(c) Mobile device located at $(1.6,1.4)$ Average Error $=0.37458 \mathrm{~m}$

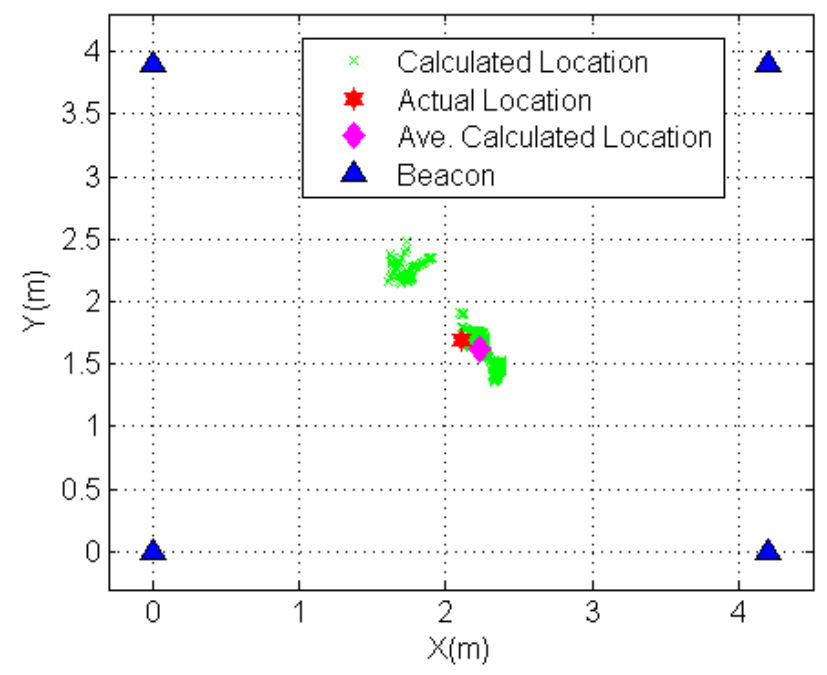

(d) Mobile device located at $(2.3,1.7)$

FIGURE V. POSITIONING RESULTS
Figure $\mathrm{V}$ shows that our algorithm achieves an average positioning accuracy of $0.2 \sim 0.4 \mathrm{~m}$. Notice that in Figure V (d), calculated locations are grouped into two clusters. The upper left cluster to the actual location is caused by unstable transmitting power of beacons.

\section{CONCLUSION}

In this paper, an algorithm is proposed for indoor positioning. The proposed algorithm first pre-processes RSSI by single-direction outlier removal and moving average. Then distance between mobile devices and beacon is calculated using pre-processed RSSI, and is filtered using Kalman filter. Finally, mobile device's location is determined by triangulation. Results show that the proposed algorithm achieves an average positioning accuracy of $0.2 \sim 0.4 \mathrm{~m}$.

\section{REFERENCE:}

[1] Zhang, Hui-Feng, Qing-Yun Du, and Chao-Fei Qiao. "Present State and Trends of the Geoinformation Industry in China." Sustainability 7.3 (2015): 2871-2884.

[2] Zhai, Di, and Zihuai Lin. "RSS-based indoor positioning with biased estimator and local geographical factor." Telecommunications (ICT), 2015 22nd International Conference on. IEEE, 2015.

[3] Yan, Kun, et al. "Current status of indoor positioning system based on visible light." Control, Automation and Systems (ICCAS), 2015 15th International Conference on. IEEE, 2015.

[4] Su, Hui-Kai, et al. "A Hybrid Indoor-Position Mechanism Based on Bluetooth and WiFi Communications for Smart Mobile Devices." Bioelectronics and Bioinformatics (ISBB), 2015 International Symposium on. IEEE, 2015.

[5] Kasantikul, Kittipong, et al. "An enhanced technique for indoor navigation system based on WIFI-RSSI." Ubiquitous and Future Networks (ICUFN), 2015 Seventh International Conference on. IEEE, 2015.

[6] Cho, Seong Yun. "A Modified Residual-based Extended Kalman Filter to Improve the Performance of WiFi RSSI-based Indoor Positioning." Journal of Institute of Control, Robotics and Systems 21.7 (2015): 684-690.

[7] Lin, Xin-Yu, et al. "A mobile indoor positioning system based on iBeacon technology." Engineering in Medicine and Biology Society (EMBC), 2015 37th Annual International Conference of the IEEE. IEEE, 2015.

[8] Palumbo, Filippo, et al. "A stigmergic approach to indoor localization using Bluetooth Low Energy beacons." Advanced Video and Signal Based Surveillance (AVSS), 2015 12th IEEE International Conference on. IEEE, 2015.

[9] Hamdoun, Safa, Abderrezak Rachedi, and Abderrahim Benslimane. "RSSI-based localisation algorithms using spatial diversity in wireless sensor networks." International Journal of $\mathrm{Ad} \mathrm{Hoc} \mathrm{and} \mathrm{Ubiquitous}$ Computing 19.3-4 (2015): 157-167.

[10] Information on http://www.aprbrother.com/en/product.htm 